(T2), $4 \mathrm{~h}(\mathrm{~T} 4)$ at $37^{\circ} \mathrm{C}$. At each time point, total viable cell counts as well as microbiome analysis through $16 \mathrm{~S}$ ribosomal shotgun sequencing will be performed and compared for differences. Bacteria isolated from each MOM will be saved, identified through 16S ribosomal sequencing and grown in culture for future studies. Fecal samples from each corresponding infant will be collected within $48 \mathrm{~h}$ after collection of the MOM sample. Stools will be homogenized and subjected to DNA extraction to perform $16 \mathrm{~S}$ ribosomal shotgun sequencing. Microbiome analysis will be conducted, compared between fecal samples as well as with the microbiome of the MOM. RESULTS/ ANTICIPATED RESULTS: Study is ongoing. We anticipate similar results with fresh or frozen MOM to that of a previous pilot study, where enriched microbiota similar to MOM was found when fresh MOM was inoculated and incubated in DHM. The microbiome analysis of the infant fecal samples may illustrate the influence that the microbiome of the MOM may have on the development of the infants' gastrointestinal microbiota. DISCUSSION/SIGNIFICANCE OF IMPACT: The purpose of this study is to provide evidence on the ability, timing, and efficacy of inoculating DHM with fresh and frozen MOM. Study results will inform future studies to support the implementation of an inoculation procedural protocol to be used in clinical practice and human milk banking. The description of the MOM microbiome, as well as the gastrointestinal microbiome, will expand scientific knowledge on the role breast milk has on the origins of health and disease.

Associations between Diabetes Mellitus and Sublingual Microvascular Disease: A Prospective Cross-Sectional Study

Nathaniel R Smilowitz ${ }^{1}$, Joseph Windheim² ${ }^{2}$ Elias Simon ${ }^{2}$ and Harmony Reynolds, MD ${ }^{3}$

${ }^{1}$ National Institutes of Health; ${ }^{2}$ New York University Langone Health and ${ }^{3} \mathrm{NYU}-\mathrm{H}+\mathrm{H}$ Clinical and Translational Science Institute

OBJECTIVES/SPECIFIC AIMS: Diabetes mellitus (DM) is a risk factor for the development of microvascular complications. We sought to determine the association between diabetes mellitus status and microvascular circulatory disease, as measured in the sublingual capillary bed. METHODS/STUDY POPULATION: We prospectively recruited adults with cardiovascular $(\mathrm{CV})$ risk factors or established CV disease, with and without DM, who were referred for invasive coronary angiography at an urban tertiary care medical center. All participants underwent non-invasive sublingual sidestream darkfield microscopy. The primary outcome was the perfused boundary region (PBR), a measure reflecting the extent to which red blood cells (RBC) penetrate the sublingual glycocalyx in vessels between 5 and $25 \mu \mathrm{m}$ in width. RESULTS/ANTICIPATED RESULTS: 57 participants were enrolled. The mean age was $66.1 \pm 11.1$ years and a majority of participants (66\%) were men. DM was present in $18(31.6 \%)$ participants. Sublingual PBR measurements were not different between participants with and without DM overall $(1.93 \mu \mathrm{m}$ vs. $1.96 \mu \mathrm{m}, \mathrm{p}=0.63)$ or in vessels with high flow $(1.48 \mu \mathrm{m}$ vs. $1.59 \mu \mathrm{m}, \mathrm{p}=0.08)$. No differences in capillary $\mathrm{RBC}$ filling $(72.9 \%$ vs $73.0 \%, \mathrm{p}=0.95)$ or perfused microvascular density ( 3112 vs. $3236 \mu \mathrm{m} / \mathrm{mm} 2, \mathrm{p}=0.32$ ) by $\mathrm{DM}$ status were observed.
DISCUSSION/SIGNIFICANCE OF IMPACT: In a population of adults with CV risk factors or disease, DM was not associated with impaired sublingual microvascular glycocalyx. Additional investigation into diabetes-induced microvascular impairment is warranted.

3067

\section{Biomechanical analysis of acute versus chronic aortic dissection flaps}

Xiaoying Lou ${ }^{1}$, Wei Sun, Fatiesa Sulejmani, Minliang Liu, Edward Chen and Bradley Leshnower

${ }^{1}$ Emory University

OBJECTIVES/SPECIFIC AIMS: Thoracic endovascular aortic repair (TEVAR) is more effective in remodeling the dissected aorta in acute versus chronic type $\mathrm{B}$ aortic dissection (TBAD). It has been hypothesized that this is due to differences in dissection flap biomechanical and structural properties but has not been confirmed in explanted human aortic tissue. We aimed to characterize and compare differences in tissue biomechanics and microstructure between acute and chronic dissection flaps that may underlie these findings. METHODS/STUDY POPULATION: Dissection flaps were obtained at time of operative repair for patients presenting for open aortic replacement to treat acute type A (ACUTE, $n=7$ ) or chronic type $\mathrm{B}$ (CHRONIC, $\mathrm{n}=7$ ) aortic dissection. Given that the current treatment modality for acute complicated TBAD is TEVAR, it was not feasible to acquire acute TBAD flaps for analysis. Tissues were cryopreserved and subjected to biaxial tensile testing in the circumferential and longitudinal directions. Stiffness was quantified by the tangent modulus (TM) in the low and high linear regions of the compiled equibiaxial response curves for each cohort. Extensibility was defined as the intersection of the fitted line from the high linear region with the $\mathrm{x}$-axis, and the degree of anisotropy (DA) was defined as the mean absolute percentage error of the strains in both directions. Flap architecture and collagen fiber organization were also compared between groups using two-photon microscopy. RESULTS/ANTICIPATED RESULTS: Average age of dissection flaps were 3.4 \pm 3.4 days in ACUTE and 1,868.7 $\pm 1,354.0$ days in CHRONIC $(\mathrm{p}=0.011)$. There were no differences in age, co-morbidities, maximum aortic diameter, and aortic wall thickness. ACUTE exhibited an anisotropic stress-strain response with increased extensibility longitudinally than circumferentially ( 0.18 vs. $0.09, \mathrm{p}=0.022, \mathrm{DA}=0.67)$ while CHRONIC demonstrated an isotropic response with similar extensibility in either direction ( 0.11 vs. $0.12, \mathrm{p}=0.606, \mathrm{DA}=0.26$ ). CHRONIC and ACUTE had comparable stiffness in the circumferential direction (TMlow 439.92 vs. 541.08, $\mathrm{p}=0.729$, and TMhigh $1585.19 \mathrm{kPa}$ vs. $1869.35 \mathrm{kPa}, \mathrm{p}=0.817)$. In the longitudinal direction, CHRONIC was significantly stiffer than ACUTE (TMhigh 8347.61 $\mathrm{kPa}$ vs. $1201.34 \mathrm{kPa}, \mathrm{p}=0.049$ ) (FIGURE). Microscopy corroborated these findings with greater collagen fiber organization circumferentially than longitudinally in ACUTE and increasing fibrosis, collagen predominance, and straightening of collagen fibers in CHRONIC. DISCUSSION/SIGNIFICANCE OF IMPACT: Compared to ACUTE, CHRONIC exhibited loss of anisotropy with increased tissue stiffness in the longitudinal direction. Increased dissection flap fibrosis and decreased compliance may explain the worse outcomes for aortic remodeling after TEVAR in chronic TBAD. This study offers biomechanical support for early TEVAR in the acute phase of uncomplicated TBAD. 